\author{
Dinara Stepaniszczewa \\ ORCID: 0000-0002-9609-4878 \\ Universität Szczecin, Szczecin
}

DOI: $10.19195 / 0435-5865.143 .22$

\title{
Konzeptuelle Metaphern in den Bezeichnungen der Teekompositionen
}

\begin{abstract}
s
In dem vorliegenden Artikel werden die konzeptuellen Metaphern in den Bezeichnungen der Teekompositionen in der polnischen, russischen und deutschen Sprache analysiert. Die theoretische Grundlage der Analyse bilden die Konzeptionen von Lakoff, Johnson (1980), Fauconnier (1985) und Fauconnier, Turner $(1998,2002)$. Es wird angestrebt, die konzeptuellen Metaphern aufzudecken, die Metaphorisierungsprozesse zu beschreiben und die durch die Teebezeichnungen aktivierten Konzeptualisierungen zu charakterisieren.
\end{abstract}

Stichwörter: konzeptuelle Metapher, mentale Räume, Blending, Teebezeichnungen

\section{Conceptual metaphors in the names of tea compositions}

Research material in this article consists of the conceptual metaphors in the names of tea varieties and tea compositions from the original data in Polish, Russian and German. The theoretical basis of the analysis is the approach by Lakoff and Johnson (1980), Fauconnier (1985), and Fauconnier and Turner $(1998,2002)$. The present study seeks to reveal conceptual metaphors, to describe the processes of metaphorization and to characterize conceptualization, activated by the tea names.

Keywords: conceptual metaphor, mental spaces, blending concept, tea names

Dinara Stepaniszczewa, Uniwersytet Szczeciński, Instytut Filologii Germańskiej, Al. Piastów 40b, bud. 5, pok. 103, 71-064 Szczecin, Polen, E-Mail: dinara_stepanishcheva@outlook.com Received: 28.09.2017, accepted: 18.05.2018 
Tee ist ein Wundermittel zur Erhaltung des Lebens und eine Kunstfertigkeit zu seiner Verlängerung.

(Myoan Eisai)

\section{Einleitung}

Eine berberische Weisheit vergleicht Tee mit der irdischen Wonne und beschreibt seinen Geschmack mit folgenden Worten: „Das erste Glas ist bitter wie das Leben, das zweite süß wie die Liebe und das dritte sanft wie der Tod“. ${ }^{1}$

Objektiv gesehen ist Tee ein Getränk, das aus Teepflanzenblättern und heißem Wasser zubereitet wird. Emotional gesehen wird Teegenuss mit Gemütlichkeit und Ruhe verbunden. ${ }^{2}$

Für den durchschnittlichen Europäer ist der Teekonsum Teil des alltäglichen Lebens. Aus einer im Jahre 2014 durchgeführten Studie geht hervor, dass weltweit pro Kopf über 726 Millionen Kilogramm Tee jährlich verbraucht werden. ${ }^{3}$ An der Spitze der Statistik befindet sich China. Die führende Position des Teekonsums in Europa gehört traditionell dem Vereinigten Königreich Großbritannien und Nordirland. Russland nimmt den 4. Rang (mit dem Indikator 3,051 kg Tee pro Einwohner im Jahr) an, Polen - den 8. (Indikator 2,204) und Deutschland - den 16. (Indikator 1,524). ${ }^{4}$ Da der Tee, laut Statistik, zu den beliebtesten Getränken in den genannten Ländern gehört, beeinflusst er auch das Verhalten der dort lebenden Menschen, was sprachlich in den Redewendungen, Sprüchen, Zitaten, Liedern und in den Konzeptualisierungen wie Geduld, Ruhe, Genuss, Zurückhaltung, Stille zum Vorschein kommt.

Als Beispiel können solche deutschen Redewendungen wie Abwarten und Tee trinken oder Tee ist Ruhe und nicht Eile dienen, in Russland ist das Sprichwort Nach dem Tee ist für die Seele Sommer populär und in einem polnischen Lied von dem bekannten Kabarettsänger Jeremi Przybora wird der Tee mit dem integralen Bestandteil glücklichen Lebens verglichen. In der hypertrophierten rhetorischen Frage „Wozu braucht man das Leben ohne Tee?“" wird die Wichtigkeit und Unverzichtbarkeit dieses Getränks scherzhaft ausgedrückt.

Der vorliegende Artikel hat zum Ziel, die Metaphorisierungs- und Blendingprozesse am Beispiel ausgewählter Bezeichnungen der Teekompositionen in der polnischen, russischen und deutschen Sprache zu beleuchten und die Vielfalt der Konzeptualisierungen, die diese Bezeichnungen hervorrufen, zu charakterisie-

${ }^{1}$ www.tea-terra.ru/wpontent/uploads/2015/10/2015_10_01_01002.png.

${ }^{2}$ www.gq-magazin.de/leben-als-mann/essen-und-trinken/tee-tee-genuss-seit-5000-jahren/teetee-in-afrika-naher-osten.

${ }^{3}$ www.priroda.su/item/3603.

${ }^{4}$ www.priroda.su/item/3606. 
ren. Die theoretische Untermauerung bilden die kognitionslinguistischen Ansätze von Lakoff, Johnson (1980) zu den konzeptuellen Metaphern und von Fauconnier (1985), Fauconnier, Turner $(1998,2002)$ zu den mentalen Räumen.

\section{Konzeptuelle Metaphern in der Theorie von Lakoff und Johnson}

Die konzeptuelle Metapher spielt eine besondere Rolle in der kognitionslinguistischen Forschung. In den Untersuchungen werden die Analysen aufgrund der konzeptuellen Metaphern aus vielfältigen Diskursen vorgestellt. Diese Tendenz beweist das wachsende Interesse an den konzeptuellen Metaphern, die den gesamten Bereich der menschlichen Erfahrung abdecken und ein bedeutendes kognitives Potenzial besitzen. Konzeptuelle Metaphern umfassen die meisten menschlichen Aktivitäten und Lebenssphären. Diese Behauptung unterstützen zahlreiche sprachwissenschaftliche Untersuchungen aus der ganzen Welt. Ihre Gesamtzahl überschreitet 2000 Publikationen (vgl. Kibrik, Kobzoewa 2002: 68).

Die Ausgangsbasis für die Weiterentwicklung der Forschung in diesem Bereich stellt nach wie vor die klassische Theorie der konzeptuellen Metaphern von Lakoff und Johnson (1980) dar. Die beiden Forscher verstehen die Metapher als Effekt der Übertragung konkreter physischer Erfahrungen auf abstrakte, auch kulturelle Phänomene (vgl. Lakoff, Johnson 2003: 27). Das Wesen der Metapher besteht darin, dass die einen Sachen oder Vorgänge mit Begriffen, die andere Sachen bzw. Vorgänge betreffen, beschrieben werden (vgl. Lakoff, Johnson 1980: 5). Die Ursprungsdomänen (source-domains) enthalten Elemente der physischen Erfahrung, und die Zieldomänen (target-domains) - abstrakte Elemente. Infolge der metaphorischen Projektion (metaphorical mapping) kommt es zur konzeptuellen Übertragung der Elemente aus den Ursprungsdomänen in die Zieldomänen. Der so erfolgende Metaphorisierungsprozess ermöglicht es, die abstrakten Erscheinungen zu begreifen (vgl. Lakoff 1993: 206). Die konzeptuellen Metaphern bilden die Grundlage des Begriffs- und Bedeutungssystems (vgl. Lakoff, Johnson 1999: 57). Die Sprache wird daher als eine wichtige Informationsquelle über das Konzeptsystem betrachtet (vgl. Lakoff, Johnson 2003: 51).

Lakoff und Johnson (1980) unterscheiden Orientierungsmetaphern, strukturelle Metaphern und ontologische Metaphern. Der Raum ist ein grundlegendes Konzept für die Bildung und Bezeichnung neuer raumloser Erfahrungen, wie es im Falle der Orientierungsmetaphern wie happy is up (Glück ist oben), sad is down (Trauer ist unten) ist (Lakoff 1990: 47). Die Orientierungsmetaphern reflektieren die Oppositionen, in denen die Erfahrung der räumlichen Orientierung in der Welt enthalten ist. Als eines der häufigsten Beispiele der strukturellen Metaphern, in denen abstrakte Begebenheiten mit Hilfe konkreter Sachverhalte ausgedrückt werden, gilt die Metapher Zeit ist Geld (Lakoff 1990: 38). Die onto- 
logischen Metaphern werden von Lakoff und Johnson als wichtiges Mittel zum Verstehen unserer Erfahrungen betrachtet. Die ontologischen Metaphern spiegeln unsere „Erfahrungen in der Welt als Objekte oder Substanzen“ wider (Feng 2010: 197). $\mathrm{Zu}$ den ontologischen Metaphern gehören auch Personifizierungen wie His religion tells him (Sein Glaube sagt ihm). Sie stützen sich auf eine besondere Art der Betrachtung der Person, weil die Äußerungen sich auf Alter, Charakter oder soziale Rollen beziehen (Lakoff, Johnson 2003: 35-45).

Von zahlreichen anderen Ansätzen zu Fragen der konzeptuellen Metaphern wie das kohärente metaphorische Modell von Spellman (1993), die Theorie der konzeptuellen Integration von Turner und Fauconnier $(1995,2000)$, die primären Metaphern von Grady (1997), Theorie der metaphorischen Modellierung von Chudinov (2003), Bindungstheorie der metaphorischen Interpretation von Ritchie (2003), deskriptorische Theorie der Metapher von Baranow und Karaulow (2008) wird im Folgenden die Theorie von Fauconnier und Turner $(1998,2002)$ näher charakterisiert, die samt der Theorie von Lakoff und Johnson (1980), wie bereits gesagt, die theoretische Grundlage der empirischen Untersuchung bildet.

\section{Mentale Räume und Blendingeffekt in der Theorie von Fauconnier und Turner}

Die Blending-Theorie von Fauconnier und Turner $(1998,2002)$ geht aus der Theorie der mentalen Räume (mental space theory) von Fauconnier (1995) hervor.

Die Theorie der mentalen Räume von Fauconnier enthält nicht nur sprachwissenschaftliche, sondern auch philosophische Bezüge auf die kognitiven Prozesse der Kommunikation. Fauconnier betont, dass die Modellierung von mentalen Räumen die Art und Weise, wie wir denken und sprechen, bestimmt (vgl. Fauconnier 1996: 7-8). Mentale Räume werden als Hilfsmittel angesehen, die von den Kommunikationsteilnehmern geschaffen werden, um die Aussagen der Gesprächspartner zu verstehen und eigene Aussagen zu bilden.

Blends werden nicht direkt aus den Komponenten der Quellräume abgeleitet: sie sind mit den ursprünglichen Räumen nicht identisch. Ein Blend leiht aus dem Quellraum nur einen Teil der Struktur. Anders als bei Lakoff, Johnson (1980), die die gegenseitige Relation zwischen zwei konzeptuellen Domänen hervorheben, betonen Fauconnier und Turner die Integration zwischen vier mentalen Räumen: zwei Eingaberäumen (input spaces), einem generischen Raum (generic space) und einem Blendingraum (blended space oder blend) (vgl. Turner, Fauconnier 1995:184). Die Anzahl der Eingaberäume kann mehr als zwei betragen. Im generischen Raum werden aufgrund der persönlichen Erfahrungen weitere Bedeutungsinhalte hinzugezogen und im Blendingraum wird anhand dieser Daten die Bedeutung aufgebaut. Dieser Prozess erfolgt dreiphasig: über die Komposition (composition), Komplettierung (completion) und Elaboration (elaboration). In 
der Phase der Komposition werden die Eingaberäume bestimmt, in der Phase der Komplettierung werden diese um die zusätzlichen Bedeutungselemente ergänzt und in den Blendingraum weitergeleitet, in dem die Phase der Elaboration erfolgt, deren Effekt die zuletzt aufgebaute Bedeutung ist (vgl. Fauconnier, Turner 1994: 314; Kowalczuk 1997: 99). Der Entstehungsprozess eines Blends lässt sich am Beispiel des Ausdrucks warmer Mantel illustrieren. Den einen Eingaberaum bildet das Adjektiv warm und den anderen das Substantiv Mantel. Im generischen Raum finden sich die Eigenschaft warm und die menschliche Empfindung der Wärme. Der Blend entsteht durch die Kompression, indem im Prozess der begrifflichen Integration die menschliche Empfindung der Wärme auf das Kleidungsstück als seine Eigenschaft übertragen wird (vgl. Libura 2007: 33-37).

Die beschriebenen Prozesse, die die Verarbeitung der Ausdrücke steuern, werden im Folgenden am Beispiel der Bezeichnungen der Teekompositionen näher beleuchtet.

\section{Teebezeichnungen und die ihnen zugrundeliegenden konzeptuellen Metaphern - eine empirische Untersuchung}

Das Untersuchungsmaterial bilden die Bezeichnungen der Teekompositionen in der polnischen, russischen und deutschen Sprache, ausgewählt aus den Online-Teekatalogen in jeder der Sprachen. Die Gesamtzahl der analysierten Teebezeichnungen beläuft sich auf 180, je 60 aus jeder der untersuchten Sprachen.

Im vorliegenden Beitrag werden 9 Teebezeichnungen aus dem ganzen Material vorgestellt, je 3 aus jeder Sprache. Die Bezeichnungen der analysierten Teekompositionen lassen sich den folgenden Kategorien zuordnen: 'Geschmackbestimmung und Duftbeschreibung', 'regional-geografische Markierungen', 'Familienwerte', 'Anspielungen auf weltbekannte Ereignisse', 'Werke und Personen', 'Zeitcharakteristika', 'Empfindungen'.

\subsection{Teebezeichnungen in der polnischen Sprache}

Analysiert werden folgende drei Teebezeichnungen: Owocowa Laguna (Obstlagune), Serce Matki (Mutterherz), Chwila przy kominku (Eine Weile am Kamin). ${ }^{5}$

\section{Owocowa Laguna (Obstlagune)}

Die Bezeichnung Owocowa Laguna (Obstlagune) entstammt den Kategorien 'Geschmackbestimmung und Duftbeschreibung' und 'regional-geografische Markierungen'. In dieser Kategorie werden Geschmacks-, Natur- und Landschafts-

\footnotetext{
${ }^{5}$ www.czasnaherbate.net/1198.html.
} 
merkmale hervorgehoben und zum Ausdruck gebracht. Bei der Bezeichnung Owocowa Laguna (Obstlagune) handelt es sich um den fruchtigen Geschmack und die exotische Landschaft. Die exotische Landschaft aktiviert zugleich die mentalen Räume um die exotischen Früchte. Der dieser Bezeichnung zugrundeliegende Metaphorisierungsprozess besteht darin, dass die konkreten Bedeutungen jeder der Komponenten dieser Bezeichnung, die den Domänen [Obst] und [exotische Landschaft] als Ursprungsdomänen entstammen, im Konzeptualisierungsprozess auf die Zieldomäne [Teegeschmack] übertragen werden. Die erste Komponente der Bezeichnung aktiviert die Konzeptualisierung des fruchtigen Geschmacks, während die zweite diese Konzeptualisierung präzisiert und auf den Geschmack exotischer Früchte wie Banane, Ananas, Kokosnuss, Papaya hinführt. Infolge dieser Konzeptualisierungsprozesse werden weitere mentale Räume z.B. um unbewohnte exotische Inseln, Karibik, Tropen, tropische Strände eröffnet.

Diese Bezeichnung der exotischen Teekomposition kann bei manchen Rezipienten die Konzeptualisierung des amerikanischen Filmdramas aus dem Jahre 1980 „Die Blaue Lagune“ aktivieren, oder auch die des Mischgetränks Cocktail Blue Lagoon. Bei der letzten wird auch die hellblaue Farbe konzeptualisiert. Der Metaphorisierungsprozess erfolgt in diesem Fall zweistufig: Zuerst wird hellblau als die Farbe des Getränks und Sirups konzeptualisiert, und dann wird diese Konzeptualisierung auf Meer und Himmel an warmen Orten übertragen (vgl. Lakoff, Johnson 1980).

\section{Serce Matki (Mutterherz)}

Die Bezeichnung Serce Matki (Mutterherz) kann sowohl der Kategorie 'Familienwerte' als auch 'Empfindungen' angehören. Relevant erscheint die graphische Form, d.h. die Großschreibung der Komponente Matki (der Mutter G.). Mit der Großschreibung der Substantive, die keine Eigennamen sind, wird in der polnischen Sprache ihre Bedeutung besonders hervorgehoben. So wird auf diese Weise metaphorisch die besondere Rolle der Mutter im menschlichen Leben zum Ausdruck gebracht und diese besonders positive Empfindung wird konzeptuell auf den Teegeschmack übertragen. Der Metaphorisierungsprozess beruht hier auf der Übertragung der konkreten Bedeutungen ihrer Komponenten Herz und Mutter aus den Domänen [Körperteile] und [Familienmitglieder] auf die Zieldomäne der [Gefühle] mit der Hervorhebung des Gefühls der Liebe, dessen Konzeptualisierung durch die possessiv gebrauchte zweite Komponente Matki zusätzlich verstärkt wird. Die Teebezeichnung aktiviert infolge dieser Prozesse positive Konzeptualisierungen, die mit den allgemein gültigen menschlichen Werten verbunden sind. Die infolge des Metaphorisierungsprozesses aufgebaute Bedeutung der Bezeichnung Mutterherz gewinnt in Bezug auf die Teekomposition Inhalte wie traditionell, gewohnt, sanft, ohne exotische, ungewöhnliche Geschmäcke und Düfte. Das Konzept Herz lässt sich auch metonymisch verarbeiten, indem es als Teil des Ganzen den Bezug auf die Person der liebenden Mutter verstärkt (vgl. Lakoff, Johnson 2003: 79-80). 


\section{Chwila przy kominku (Eine Weile am Kamin)}

An der Bezeichnung Chwila przy kominku (Eine Weile am Kamin) lässt sich der Blendingeffekt verfolgen. Als Eingaberäume können in der Kompositionsphase [Zeitangaben], aktiviert durch die Komponente Weile und [Räume], aktiviert durch die Komponente am Kamin bestimmt werden. In der Komplettierungsphase kommen im generischen Raum infolge der Verschmelzung der durch die beiden Komponenten ausgelösten Bedeutungsinhalte die Wärme als Element der Domäne [Empfindungen] und ähnliche positive Elemente aus der Domäne [Gefühle] wie angenehm und gemütlich auf. In der Evaluationsphase werden diese integriert. Dabei wird mit der Weile eine nicht präzisierte, aber angenehme Zeitdauer konzeptualisiert und das hervorgerufene angenehme Gefühl wird durch die Ortsangabe am Kamin zusätzlich verstärkt, indem sie die mentalen Räume um Kaminfeuer, Wärme und Gemütlichkeit eröffnet.

Diese Teebezeichnung kann ebenfalls Konzeptualisierungen wie Herbst- und Winterzeit, Weihnachten, Winterferien, Gebirge aktivieren, die wiederum mentale Räume um Kälte und Dunkelheit, aber auch Wintersportaktivitäten eröffnen können. Diese bleiben aber im Hintergrund der positiven Konzeptualisierung einer gemütlichen Zeit am Kaminfeuer. Wird dabei das Zuhause konzeptualisiert, können sich mentale Räume um ognisko domowe (Haus und Herd) eröffnen, die mit weiteren Konzeptualisierungen wie Familienglück, Familienwärme, Liebe, Vertrauen, Freude und Ruhe einhergehen. Diese Konzeptualisierungen erfassen auch greifbare Objekte und Umgebungsvorstellungen: Gegenstände, Dekorationen, Geschenke, Landschaften. Alle Konzeptualisierungen beziehen sich auf positive zwischenmenschliche Kontakte.

Laut Fauconnier und Turner treten bei der Metaphorisierung komplexe dynamische Integrationsprozesse ein, infolge deren bei den Rezipienten neue mentale Räume entstehen. Sie enthalten Elemente aus den Quelldomänen und Zieldomänen und stellen Mischgebilde dar. Dabei kennzeichnen sie auch völlig neue Eigenschaften. Dieser Sachverhalt führt zu einer wesentlichen Umwandlung der Bedeutungsstruktur, so dass neue mentale Räume, Blends, entstehen (vgl. Fauconnier, Turner 1994, 1998; Turner, Fauconnier 1995, 2002). Die infolge der Blendingprozesse entstehende Bedeutung der Bezeichnung Eine Weile am Kamin enthält in Bezug auf die Teekompositionen Inhalte wie erwärmend, gewürzt, duftig, mit Zimt, Ingwer, Gewürznelke in der Herbst- und Winterzeit.

\subsection{Teebezeichnungen in der russischen Sprache}

Analysiert werden folgende drei Teebezeichnungen: Белье ночи (Weiße Nächte), 12 подвигов Геркулеса (Die 12 Arbeiten des Herkules), Сибирская красавица (Sibirische Schöne). ${ }^{6}$

\footnotetext{
${ }^{6}$ www.chay.info/catalog/chay/zelenyy-chay-s-dobavkami.
} 
Белье ночи (Weiße Nächte)

Die Bezeichnung Белье ночи (Weiße Nächte) gehört in die Kategorie 'Zeitcharakteristika'. Der Metaphorisierungsprozess besteht in der Übertragung der konkreten Bedeutung der Farbe wei $\beta$ und der Tageszeit Nacht auf die Jahreszeit vom Ende Mai bis Mitte Juli in Sankt-Petersburg. Konzeptualisiert wird dabei die Erscheinung der weißen Nächte selbst. Zugleich kann die Bezeichnung, als eigenartiges Symbol von Sankt Petersburg, besonders bei den russischsprachigen Rezipienten, mit dieser Stadt in Verbindung gebracht werden. In Sankt Petersburg wird diese Bezeichnung popularisiert und kultiviert. Zu dieser Jahreszeit finden dort massenhafte Veranstaltungen, Feste, Konzerte und Ausstellungen statt.

Bei den russischsprachigen Rezipienten kann sie auch die Konzeptualisierung des Stadtgründungstages, des 27. Mai 1703 aktivieren. Die Weißen Nächte von Sankt Petersburg sind auch dank der gleichnamigen Erzählung von Dostojewski aus dem Jahre 1848 weltbekannt, so dass auch bei ausländischen Rezipienten die Bezeichnung Weiße Nächte die Konzeptualisierung dieser russischen Stadt aktivieren kann.

Die deutschsprachigen Rezipienten können diese Bezeichnung mit der historischen Familiensaga „Weiße Nächte, weites Land“ von Martina Sahler (2013) über die Auswanderung der Deutschen nach Sankt Petersburg im 18. Jahrhundert verbinden. Bei ihnen können Konzeptualisierungen wie Russland, Sommerzeit, Nordlicht, Finnischer Meerbusen durch diese Bezeichnung hervorgerufen werden.

Laut Lakoff und Johnson (1980) kann der Ausdruck die Nächte in dieser Metapher nicht nur zeitorientierte, sondern auch räumliche Eigenschaften ausdrücken, so dass diese Metapher der Unterkategorie der räumlichen ontologischen Metaphern zugeordnet werden kann.

Die Bezeichnung stellt zugleich ein mehrdeutiges Oxymoron dar. Die eine erfahrungsmäßig konzeptualisierte Opposition betrifft die Unterscheidung zwischen hell und dunkel sowie Tag und Nacht, und die andere bezieht sich auf die klimageografischen Aspekte und die Unterscheidung zwischen Nord und Süd sowie kalt und warm. Die Erscheinung der

Weißen Nächte findet in den Sommermonaten statt und ist auch unter der Bezeichnung der Polartage bekannt. Als Naturerscheinung sind weiße Nächte nur in dem geografischen Raum um den Polarkreis zu beobachten. Das Oxymoron ergibt sich aus der konzeptuellen Integration der widersprüchlichen Erfahrungen der gewöhnlich als warm konzeptualisierten Sommerzeit und der Kälte des Polarkreises. Im Allgemeinen kann die Bezeichnung weiße Nächte die Vorstellung der skandinavischen Landschaften hervorrufen.

Weiße Nächte als Teebezeichnung können daher vielfältig konzeptualisiert und sowohl auf die Metaphorisierungs- als auch Blendingprozesse zurückgeführt werden. Die Bezeichnung kann in Bezug auf den Tee mentale Räume um helle Farben und milden Geschmack des Teegetränks eröffnen. 


\section{2 подвигов Геркулеса (Die 12 Arbeiten des Herkules)}

Die Bezeichnung 12 подвигов Геркулеса (Die 12 Arbeiten des Herkules) gehört in die Kategorie 'Anspielungen auf weltbekannte Ereignisse, Werke und Personen'. Der Metaphorisierungsprozess besteht in der Übertragung der Eigenschaften, die mit der mythologischen Gestalt des Halbgottes Herkules verbunden sind, auf die Teemerkmale. Neben den Konzeptualisierungen aus den Domänen [griechische und römische Mythologie] und [Antike] u.a. den Hauptaufgaben von Herakles, dem Nemeischen Löwen, Hydra (Lernäischen Schlange), der Kerynitischen Hirschkuh, dem Stymphalischen Vogel, der Rosse des Diomedes, der Amazonenkönigin Hippolyte, dem Apfel der Hesperiden werden die Eigenschaften wie Stärke und Mut durch diese Bezeichnung hervorgerufen. 12 подвигов Геркулеса (Die 12 Arbeiten des Herkules) aktivieren als Teebezeichnung die Konzeptualisierung einer aus 12 verschiedenen Bestandteilen: Sorten, Aromen, Früchten, Kräutern hergestellten Teekomposition mit stärkender und ermutigender Wirkung.

\section{Сибирская красавица (Sibirische Schöne)}

Die Bezeichnung Сибирская красавиия (Sibirische Schöne) gehört in die Kategorie 'regional-geografische Markierungen'. Die erste Komponente der Bezeichnung aktiviert die Konzeptualisierungen der Witterungsbedingungen und des ewigen Schnees. Sibirien spielt eine bedeutende Rolle für Russland nicht nur als wichtiges Erdöl-und Holzindustriegebiet. Diese Region ist weltweit als spezifisches Kennzeichen des ganzen Landes bekannt. Sibirien ist ein berühmtes Reiseziel für viele Touristen. Dieses geografische Gebiet wird stereotypisch und autostereotypisch vielfältig konzeptualisiert, u.a. mit Schnee, Kälte, mit der Transsibirischen Eisenbahn. Die zweite Komponente aktiviert die Konzeptualisierung einer gut beseelten Person und eröffnet mentale Räume um Ästhetik, Attraktivität und andere positive Eigenschaften einer schönen Frau. Diese Konzeptualisierung wird durch die konzeptuellen Inhalte der ersten Komponente präzisiert, die bei den russischsprachigen Rezipienten die Konzeptualisierung eines slawischen Mädchens mit blauen Augen und blonden Haaren aktiviert und die Vorstellungen der Kräuter, Beeren und Aromapflanzen aus der Taiga hervorruft.

Der Name dieser Teekomposition kann als Effekt des Blendingprozesses angesehen werden. Aus den zwei Eingaberäumen (das Adjektiv sibirische und das Substantiv Schöne) kommen in den generischen Raum Bedeutungsinhalte, wie Schnee, Kälte, Russland und Schönheit, Weiblichkeit, Attraktivität hinein. Hinzu kommt die Vorstellung einer Frau. Im Blendingraum wird die Gestalt eines hellhaarigen, helläugigen, attraktiven, jungen Mädchens mit slawischen Gesichtszügen aus der nördlichen Region Russlands konzeptualisiert. 


\subsection{Teebezeichnungen in der deutschen Sprache}

Analysiert werden folgende drei Teebezeichnungen: Ostfriesen Sonntagstee, Süßer Drachenkuss, Sanfter Weckruf. ${ }^{7}$

\section{Ostfriesen Sonntagstee}

Die Bezeichnung Ostfriesen Sonntagstee gehört in die Kategorie der 'regional-geografischen Markierungen'. Sie aktiviert Elemente aus den Domänen [Erdkunde], [Ethnien], [Zeit] und [Raum] mit Konzeptualisierungen wie Niedersachen, Nordwesten, Nordsee, Küste und Städte, z.B. Emden, Aurich, Leer, Norden, Wittmund, Plattdeutsch (u.a. Moin, Platt), Klootschießen, Teekultur, Teebräuche und Teekonsum, Sonntag, Feiertag, letzter Tag der Woche, Gottesdient. Zu den Bräuchen gehört u.a. Dree is Oostfresenrecht (Drei ist Ostfriesenrecht). In der Tradition wird der Tee als Begrüßungsgetränk betrachtet und die Gäste werden aufgefordert, mindestens drei Tassen Tee zu trinken, wenn sie die Gastgeber nicht verletzen wollen. In den Bedeutungsinhalten dieser Teebezeichnung spiegelt sich die Tradition des Teekonsums aus der ostfrischen Region, verbunden mit der höchsten Teequalität wider.

\section{Süßer Drachenkuss}

An der Bezeichnung Süßer Drachenkuss lässt sich wieder der Blendingprozess verfolgen. Als Eingaberäume lassen sich [Geschmäcke], aktiviert durch die Komponente sü $\beta$, [Gefahren], aktiviert durch die Komponente Drache und [Gefühle], aktiviert durch die Komponente Kuss bestimmen. Diese drei Eingaberäume führen dazu, dass im generischen Raum infolge des Komplettierungsprozesses weitere Bedeutungsinhalte aus den genannten mentalen Räumen aufkommen, wie Zucker, Liebe, Fliegen, Feuer, Angst und Tod, Leidenschaft und Zärtlichkeit. Die Teebezeichnung stellt ein Oxymoron dar und die in ihr enthaltene Widersprüchlichkeit erhöht das Interesse am Geschmack dieses Tees. Die Wirkung der negativ konzeptualisierten Bedeutungsinhalte der Komponente Drache wird durch die positiv konzeptualisierten Bedeutungsinhalte der Komponenten süß und Kuss abgeschwächt.

Es muss hinzugefügt werden, dass die Komponente Drache auch Hinweise auf die chinesische Kultur hervorrufen kann, in der dieses mythologische Wesen keine negative Rolle spielt, so dass im Endeffekt des Elaborationsprozesses diese Teebezeichnung Bedeutungsinhalte um Teequalität, Teetraditionen, Teezeremonien, süßlichen, intensiven, grünen Tee verknüpfen kann.

\section{Sanfter Weckruf}

Die Bezeichnung Sanfter Weckruf gehört in die Kategorie der 'Empfindungen'. Die erste Komponente aktiviert Elemente aus der Domäne [Eigenschaften] und die zweite aus der Domäne der [Tätigkeiten]. Der Metaphorisierungsprozess besteht

\footnotetext{
${ }^{7}$ www.teaworld.de/catalogsearch/result/?q=saft.
} 
in der Übertragung der konkreten Bedeutungen der beiden Komponenten auf die Merkmale der Teekomposition. Durch das Adjektiv sanft werden als Tageszeit des Weckrufs die frühen Morgenstunden aktiviert, wenngleich auch andere Tageszeiten konzeptualisiert werden können.

Die Bezeichnung kann sowohl positive als auch negative Konzeptualisierungen hervorrufen, von der konkreten Situation, dem Gesundheitszustand, den persönlichen Erfahrungen und Gewohnheiten abhängig. Die Komponente Weckruf kann mentale Räume um unangenehme Töne eröffnen, die jedoch gemildert werden, da das Adjektiv sanft positive Konzeptualisierungen aktiviert. So wird mit dem Weckruf konzeptuell ein angenehmes Erwachen verbunden, das in den Morgenstunden Konzeptualisierungen wie Sonnenaufgang, Morgen, Aufbruch eines neuen Tages, sonnig, fröhlich, ruhig, harmonisch aktivieren kann.

\section{Fazit}

Die Analyse der konzeptuellen Metaphern, die den Teebezeichnungen zugrunde liegen, zeigt, dass infolge der Metaphorisierungsprozesse die konkreten Bedeutungen der Komponenten dieser Bezeichnungen auf abstrakte Aspekte der Teemerkmale und ihrer Auswirkungen auf den Menschen übertragen werden. Zu den Zieldomänen gehören u.a. [Geschmäcke], [Eigenschaften] und [Empfindungen].

Die Bedeutungen der Teekompositionen stellen nicht selten einen Blendingeffekt dar. Unter den sich eröffnenden mentalen Räumen finden sich u.a. Ortschaften, Landschaften, Räumlichkeiten, Bräuche, Jahreszeiten, Tätigkeiten und Sachen.

Die Datenanalyse zeigt, wie inhaltsreich die Bedeutungen der Bezeichnungen der Teekompositionen sein können, je nachdem, welche Elemente aus welchen Domänen und mentalen Räumen aktiviert werden.

\section{Literatur}

Adams, John Charles (1990): Linguistic Values and Religious Experiences: An Analysis of Clothing Metaphors in Alexander Richardson's Ramist-Puritan Lectures on Speech, Speech is a garment to cloath our reason. In: Quarterly Journal of Speech 76. S. 58-68.

Baldauf, Christa (1997): Metapher und Kognition. Grundlagen einer neuen Theorie der Alltagsmetapher. Frankfurt am Main.

Baranow, Anatoliy / Karaulow, Jurij (2008): Kompatibilität von metaphorischen Modellen. In: Fragen der Sprachwissenschaft 2. S. 73-94.

Budaev, Eduard / Chudinov, Analotiy (2008): Metaphern in der politischen Kommunikation. Moskau.

Chudinow, Waleriy (2003): Metaphorisches Mosaik in der modernen politischen Kommunikation. Jekaterinburg. 
Feng, Xiaohu (2010): Konzeptuelle Metaphern als textkohärenzstiftendes Instrument. Beijing.

Kibrik, Andrej / Kobzoewa, Irina (2002): Fundamental Directions in American Linguistics. Moskau.

Kowalczuk, Lidia (1997): Theory of conceptual blending and conceptual integration von Faconnier and Turner. In: Questions of Philology 6. S. 99.

Kudla, Martin (2008): Die Konzeptualisierung von Politik als Krieg - Metaphern in der politischen Berichterstattung, In: SASI Heft 12. S. 31.

Lakoff, George (1990): The Invariance Hypothesis: Is Abstract Reason Based on Image Schemata? In: Cognitive Linguistics 1(2). S. 257-266.

Lakoff, George (1991): Metaphor and War. The Metaphor System Used to Justify War in the Gulf. URL: http://metaphor.uoregon.edu/lakoff-1.htm (Zugriff am 17.06.2017).

Lakoff, George (1993): The Contemporary Theory of Metaphor. In: Ortony, Andrew (Hrsg.): Metaphor and Thought. Cambridge. S. 202-251.

Lakoff, George (1995): Metaphor, morality, and politics, or, why conservatives have left liberals in the dust. URL: http://www.wwcd.org/issues/Lakoff.html (Zugriff am 22.09.2017).

Lakoff, George / Johnson, Mark (1980): Metaphors We Live by. Chicago.

Lakoff, George / Johnson, Mark (1999): Philosophy in the flesh. New York.

Lakoff, George / Johnson, Mark (2003): Leben in Metaphern. Konstruktion und Gebrauch von Sprachbildern. Heidelberg.

Libura, Agnieszka (2007): Amalgamaty kognitywne w sztuce. Kraków.

Ritchie, David (2003): Argument is war or is it a Game of Chess? Multiple Meanings in the Analysis of Implicit Metaphors. In: Metaphor and Symbol 18 (2). S. 125-146.

Ritchie, David (2004): Common Ground in Metaphor Theory: Continuing the Conversation. In: Metapher and Symbol 19 (3). S. 233-244.

Schmitt, Rudolf (2004): Review Essay: Diskussion ist Krieg, Liebe ist eine Reise, und die qualitative Forschung braucht eine Brille. Heidelberg. URL: http://www.ssoar.info/ssoar/handle/ document/9337 (Zugriff am 21.09.2017).

Schnadwinkel, Birte (2002) : Neue Medien - neue Metaphern? Sprachliche Erschließung des neuen Mediums Internet durch Metaphern (deutsch-französisch). Oldenburg.

Skrebtsova, Tatiana (2000): American School of Cognitive Linguistics. Sankt Petersburg.

Spellman, Barbara / Ullman, Jodie / Holyoak, Keith (1993): A Coherence Model of Cognitive Consistency. Dynamics of Attitude Change During the Persian Gulf War. In: Journal of Social Issues 49 (4). S. 147-165.

Turner, Mark (1990): Aspects of the Invariance Hypothesis. In: Cognitive Linguistics 1(2). S. 247-255.

Turner, Mark / Fauconnier, Gilles (1995): Conceptual Integration and Formal Expression. In: Metaphor and Symbolic Activity 10(3). S. 183-204.

Turner, Mark / Fauconnier, Gilles (2000): Metaphor, Metonymy, and Binding, Metaphor and Metonymy at the Crossroads: A Cognitive Perspective. New York.

\section{Quellen}

www.chay.info/catalog/chay/zelenyy-chay-s-dobavkami (Zugriff am 12.05.2017).

www.czasnaherbate.net/1198.html (Zugriff am 10.05.2017).

www.gq-magazin.de/leben-als-mann/essen-und-trinken/tee-tee-genuss-seit-5000-jahren/tee-tee-inafrika-naher-osten (Zugriff am 4.08.2017).

www.priroda.su/item/3603 (Zugriff am 21.09.2017).

www.tea-terra.ru/wpontent/uploads/2015/10/2015_10_01_01002.png (Zugriff am 3.08.2017).

www.teaworld.de/catalogsearch/result/?q=saft (Zugriff am 10.05.2017). 